\title{
NON-EUCLIDEAN GEOMETRY AND DIFFERENTIAL EQUATIONS
}

\author{
A. G. POPOV \\ Chair of Mathematics, Department of Physics, Moscow State University \\ 119899 Moscow, Russia \\ E-mail: popov@ap.phys.msu.su
}

\begin{abstract}
In this paper a geometrical link between partial differential equations (PDE) and special coordinate nets on two-dimensional smooth manifolds with the a priori given curvature is suggested. The notion of G-class (the Gauss class) of differential equations admitting such an interpretation is introduced. The perspective of this approach is the possibility of applying the instruments and methods of non-Euclidean geometry to the investigation of differential equations.

The equations generated by the coordinate nets on the Lobachevsky plane $\Lambda^{2}$ (the hyperbolic plane) take a particular place in this study. These include sine-Gordon, Korteweg-de Vries, Burgers, Liouville and other equations. They form the so-called $\Lambda^{2}$-class (the Lobachevsky class). The theorems on the mutual transformation of solutions of $\Lambda^{2}$-class equations are formulated.

On the base of the developed approach a transformation allowing one to construct global solutions of Liouville type equations from solutions of the Laplace equation is established. Natural generalizations of the well-known nonlinear PDE from the non-Euclidean geometry point of view are proposed. The possibility of the applications of the discussed formalism in the phase spaces theory is stressed.
\end{abstract}

1. The Gauss formula as the generalized differential equation. On the plane of parameters $(x, t)$ consider the following differential quadric quantity:

$$
d s^{2}=E d x^{2}+2 F d x d t+G d t^{2},
$$

with coefficients

$$
E=E[u], \quad F=F[u], \quad G=G[u] .
$$

depending upon the unknown function $u(x, t)$ and its derivatives.

1991 Mathematics Subject Classification: 35Q53.

This work was supported by the Russian Foundation of Fundamental Research (grant 93011-16076).

The paper is in final form and no version of it will be published elsewhere. 
Let us calculate the curvature $K$ of the form (1) according to the Gauss formula [17]:

$$
K=-\frac{1}{4 W^{2}} \operatorname{det}\left|\begin{array}{lll}
E & E_{x} & E_{t} \\
F & F_{x} & F_{t} \\
G & G_{x} & G_{t}
\end{array}\right|-\frac{1}{2 \sqrt{W}}\left[\frac{\partial}{\partial t} \frac{E_{t}-F_{x}}{\sqrt{W}}-\frac{\partial}{\partial x} \frac{F_{t}-G_{x}}{\sqrt{W}}\right],
$$

where $W=E G-F^{2}$.

The right side of (3) is the known expression for the Gauss curvature $K$ through the coefficients $E, F, G$ and their derivatives with respect to $x, t$ (up to the second order). Supposing that the curvature $K=K(x, t)$ is given beforehand, one can interpret the relation (3) as a partial differential equation for $u(x, t)$ :

$$
\mathcal{F}[u(x, t)]=0 .
$$

That is, if $u(x, t)$ is a solution of (4), the form (1) defines in the $(x, t)$ plane (or on the two-dimensional smooth manifolds) a metric with the linear element (1) and with the a priori given Gaussian curvature $K=K(x, t)$. Or vice versa, the differential form (1) with the beforehand given curvature $K(x, t)$ generates (by means of the Gauss formula (3)) the differential equation (4) for the unknown function $u(x, t)$.

The following examples will help us to illustrate this interpretation.

1. Consider

$$
d s^{2}=d x^{2}+2 \cos u(x, t) d x d t+d t^{2} .
$$

In this case the coefficients (2) are chosen as follows:

$$
E \equiv 1, \quad F=\cos u(x, t), \quad G \equiv 1 .
$$

Calculating the Gauss curvature $K(x, t)$ of the form (5) by means of formula (3) we obtain the equation

$$
u_{x t}=-K(x, t) \sin u \text {. }
$$

Relation (6) was originally obtained by P. L. Tchebychef [23] in 1878 in his investigation of special nets of lines on surfaces (at present such nets are called Tchebychef nets). A Tchebychef net has equal opposite sides in any of its coordinate quadrangles. The $u(x, t)$ of the Tchebychef equation (6) has the explicit geometrical meaning of the net angle. In case $K(x, t) \equiv-1$ (curvature of Lobachevsky plane $\Lambda^{2}$ ) the equation (6) transforms into the well-known sine-Gordon equation:

$$
u_{x t}=\sin u \text {. }
$$

It should be pointed out that every regular solution $u(x, t)$ of the sine-Gordon equation (7) has the meaning of the net angle of a Tchebychef net on the corresponding part of the Lobachevsky plane $\Lambda^{2}$.

2. Set

$$
d s^{2}=\eta^{2} d x^{2}+2 \eta\left(\frac{\eta u^{2}}{2}+\eta^{3}\right) d x d t+\left(\eta^{2} u_{x}^{2}+\left(\frac{\eta u^{2}}{2}+\eta^{3}\right)^{2}\right) d t^{2}
$$

In this case

$$
E=\eta^{2}, \quad F=\eta\left(\eta \frac{u^{2}}{2}+\eta^{3}\right), \quad G=\eta^{2} u_{x}^{2}+\left(\eta \frac{u^{2}}{2}+\eta^{3}\right)^{2} .
$$


Supposing $K(x, t) \equiv-1$ (Lobachevsky plane $\Lambda^{2}$ ), we obtain for the metric (8) the following equation (of type (4)):

$$
u_{t}=\frac{3}{2} u^{2} u_{x}+u_{x x x}
$$

the so-called modified Korteweg-de Vries equation (MKdV). MKdV is also defined by its own coordinate net on the Lobachevsky plane $\Lambda^{2}$. This net can naturally be called a MKdV-net.

Originally a similar geometrical interpretation of $\mathrm{KdV}$ and $\mathrm{MKdV}$ equations was obtained in $[20,21]$.

3. For

or

$$
d s^{2}=\frac{e^{u}}{2}\left(d x^{2}+d t^{2}\right)
$$

under the condition $K \equiv-1$ we get the equation

$$
E=\frac{e^{u}}{2}, \quad F=0, \quad G=\frac{e^{u}}{2}
$$

$$
\Delta_{2} u=e^{u}, \quad \Delta_{2}=\frac{\partial^{2}}{\partial x^{2}}+\frac{\partial^{2}}{\partial t^{2}}
$$

the elliptic Liouville equation. If $u(x, t)$ is a solution of $(11)$, the net $(x, t)$ (the Liouville net) with the linear element (10) arises on $\Lambda^{2}$.

The examples mentioned above show that well-known nonlinear equations are generated by metrics of a special type with given curvature. Nonlinearity of arising equations along with possible nonlinearity of the coefficients $E, F, G$ from (2) is stipulated first of all by the nonlinearity of the discriminant $W=E G-F^{2}$ of the metric (1) in formula (3).

\section{Basic concepts and theorems}

Definition 1. A partial differential equation belongs to the $\mathbf{G}$-class (Gauss class) or is called a $\mathbf{G}$-equation if it is generated by a metric of type (1) with a given Gauss curvature $K(x, t)$ in the way mentioned above (by means of the Gauss formula).

The curvature of the generating metric is of fundamental significance when the differential equation from the $\mathbf{G}$-class is under study. In this connection the equations generated by metrics of constant curvature are of particular interest for the geometrical investigation. In this case the curvature of the generating metric has the meaning of an invariant, fixed under possible transformations of the corresponding coordinate nets.

It turns out that a great number of well-known nonlinear differential equations are generated by metrics of curvature $K(x, t) \equiv-1$ (the curvature of the Lobachevsky plane $\left.\Lambda^{2}\right)$.

Definition 2. A differential equation is called a $\Lambda^{2}$-equation (or belongs to the $\Lambda^{2}$ class, Lobachevsky class) if it is generated by a metric of Gauss curvature $K \equiv-1$.

Metrics of curvature $K \equiv-1$ are also called pseudospherical metrics.

Membership of different equations in the $\Lambda^{2}$-class results in local equivalence of their solutions. The following theorems deal with the transformation of local solutions of $\Lambda^{2}$ equations. 
TheOREM 1 ([8, 16]). Assume that two different analytic differential equations belong to the $\Lambda^{2}$-class. Then from a local analytic solution of one of the equations one can always construct a local solution of the second one and vice versa.

For example for the sine-Gordon equation theorem 1 has the following content.

THEOREM 2 ([13, 16]). Assume that some analytic differential equation

$$
\mathcal{F}[u(x, t)]=0
$$

belongs to the $\Lambda^{2}$-class. Then from any local analytic solution $u(x, t)$ of this equation one can always construct a local analytic solution of the sine-Gordon equation

$$
z_{\tilde{x} \tilde{y}}=\sin z(\tilde{x}, \tilde{y})
$$

using the formula

$$
\cos z=\left.\left[\frac{\partial f_{1}}{\partial \tilde{x}} \frac{\partial f_{1}}{\partial \tilde{y}} E+\left(\frac{\partial f_{1}}{\partial \tilde{x}} \frac{\partial f_{2}}{\partial \tilde{y}}+\frac{\partial f_{1}}{\partial \tilde{y}} \frac{\partial f_{2}}{\partial \tilde{x}}\right) F+\frac{\partial f_{2}}{\partial \tilde{x}} \frac{\partial f_{2}}{\partial \tilde{y}} G\right]\right|_{\substack{x=f_{1}(\tilde{x}, \tilde{y}) \\ t=f_{2}(\tilde{x}, \tilde{y})}},
$$

where $E=E[u(x, t)], F=F[u(x, t)]$ and $G=G[u(x, t)]$ are the coefficients of the pseudospherical metric generating equation (12).

In addition the functions $f_{1}, f_{2}$ from (13) satisfy

$$
\frac{\partial^{2} f_{\gamma}}{\partial \tilde{x} \partial \tilde{y}}+\Gamma_{\alpha \beta}^{\gamma} \frac{\partial f_{\alpha}}{\partial \tilde{x}} \frac{\partial f_{\beta}}{\partial \tilde{y}}=0, \quad \gamma=1,2,
$$

where $\Gamma_{\alpha \beta}^{\gamma}$ are the Christoffel symbols of the pseudospherical metric of type (1) generating the equation (12) and written in the variables $x \equiv f_{1}, t \equiv f_{2}$, i.e. $\Gamma_{\alpha \beta}^{\gamma}=\Gamma_{\alpha \beta}^{\gamma}\left(f_{1}, f_{2}\right)$.

It should be pointed out that the arbitrariness in choosing the local transformation from the net $(x, t)$ to the net $(\tilde{x}, \tilde{t})$ in theorem 2 is characterized by the choice of the initial conditions for constructing the local Tchebychef net $(\tilde{x}, \tilde{t})$.

The transformation established in theorems 1,2 is connected with a change of independent variables and from the geometrical point of view has the meaning of a transformation from one coordinate net to another on the plane $\Lambda^{2}$.

In the next section it will be shown that the suggested geometrical interpretation of differential equations is effective not only for local but also for global solutions.

\section{Geometrical methods for construction of global solutions of some non- linear equations}

3.1. Construction of exact solutions of the elliptic Liouville equation from solutions of the Laplace equation. We now obtain a transformation connecting the solutions of two elliptic equations: the Liouville equation

$$
\Delta_{2} u=e^{u}, \quad \Delta_{2}=\frac{\partial^{2}}{\partial x^{2}}+\frac{\partial^{2}}{\partial t^{2}}
$$

and the Laplace equation

$$
\Delta_{2} v=0 .
$$

THEOREM 3. From any solution $v(x, t) \neq$ const of the Laplace equation (15) one can construct three types of solutions $u(x, t)$ of the elliptic Liouville equation (11) using the 
formulas:

$$
\begin{aligned}
& \text { 1. } u(x, t)=\ln \frac{2\left(v_{x}^{2}+v_{t}^{2}\right)}{v^{2}}, \\
& \text { 2. } u(x, t)=\ln \frac{2\left(v_{x}^{2}+v_{t}^{2}\right)}{\operatorname{sh}^{2} v}, \\
& \text { 3. } u(x, t)=\ln \frac{2\left(v_{x}^{2}+v_{t}^{2}\right)}{\sin ^{2} v} .
\end{aligned}
$$

Proof. To construct solutions of (11) we consider the auxiliary $\Lambda^{2}$-equation

$$
y_{\tau \tau}-y=0, \quad y=y(\tau)
$$

(an ordinary differential equation), which is generated by the metric

$$
d s^{2}=y^{2}(\tau) d \chi^{2}+d \tau^{2}, \quad K(\chi, \tau) \equiv-1 .
$$

Note that the metric (10) generating the equation (11) defines the isothermic coordinate net and the linear element (20) is written in the semigeodesic coordinate net.

Let us realize the transformation from the semigeodesic coordinate net $(\chi, \tau)$ to the isothermic coordinate net $(x, t)$ (the Liouville net) by the change of variables

$$
w(x, t)=\chi, \quad v(x, t)=\int \frac{d \tau}{y(\tau)} .
$$

The substitution (21) transforms the metric (20) to the form (10) if

$$
v_{x}^{2}+w_{x}^{2}=v_{t}^{2}+w_{t}^{2}, \quad v_{x} v_{t}+w_{x} w_{t}=0 .
$$

In addition a solution $u(x, t)$ of (11) is defined by

$$
u(x, t)=\ln \left[2 y^{2}(\tau(x, t)) \cdot\left(v_{x}^{2}+w_{x}^{2}\right)\right] .
$$

The system (22) links two arbitrary harmonically conjugate functions $v$ and $w$ satisfying the Cauchy-Riemann conditions

$$
v_{x}=w_{t}, \quad v_{t}=-w_{x},
$$

and consequently the Laplace equation

$$
\Delta_{2} v=0, \quad \Delta_{2} w=0 .
$$

Let us construct a solution $u(x, t)$ of the equation (11) in accordance with (23). For that purpose we write the metric (20) in case of the general solution

$$
y(\tau)=c_{1} e^{\tau}+c_{2} e^{-\tau}, \quad c_{1}, c_{2}=\text { const },
$$

of equation (19). We choose the solution $y(\tau)(25)$ (the constants $c_{1}, c_{2}$ are fixed) and transform the metric (20) to the form (10) by the change of variables (21). In this case using the relation (21) for the given $y(\tau)$ we find a relation $\tau=\tau(x, t)=\tau(v(x, t))$ necessary for the formula (23) to hold. Depending on the signs of $c_{1}$ and $c_{2}$ in (25) the 
relation $\tau=\tau(v)$ and consequently $y^{2}(\tau)$ will take one of the following three forms:

1. $y^{2}(\tau(v))=\frac{1}{v^{2}}$,

2. $y^{2}(\tau(v))=\frac{1}{\operatorname{sh}^{2} v}$,

3. $y^{2}(\tau(v))=\frac{1}{\sin ^{2} v}$.

The substitution of (26) in (23) (taking into account (24)) gives (16)-(18). Theorem 3 is proved.

Remark 1. The validity of (16)-(18) can be verified by direct substitution in the Liouville equation (11). In this case the following statement is useful: if $v^{(k)}(x, t) \neq$ const is a solution of the Laplace equation (15) then the function $v^{(k+1)}(x, t)$ :

$$
v^{(k+1)}=\ln \left(v_{x}^{(k)^{2}}+v_{t}^{(k)^{2}}\right)
$$

is also a solution of this equation.

The formula stated above can be regarded as the Bäcklund autotransformation for the Laplace equation.

Remark 2. From the above considerations and in particular from the relation (24) one can also make the conclusion: given any analytic function of the complex variables $f(z)=v(x, t)+i w(x, t)$ one can always construct by using the formulas (16)-(18) three types of solutions of the Liouville equation.

It should be noted that the solutions (16)-(18) of the Laplace equation may be also written in the gradient form (convenient, for example, for the numerical integration of the equations):

$$
\begin{aligned}
& \text { 1. } u(x, t)=\ln \left[2(\operatorname{grad}(\ln v))^{2}\right], \\
& \text { 2. } u(x, t)=\ln \left[2\left(\operatorname{grad}\left(\ln \operatorname{th} \frac{v}{2}\right)\right)^{2}\right], \\
& \text { 3. } u(x, t)=\ln \left[2\left(\operatorname{grad}\left(\ln \tan \frac{v}{2}\right)\right)^{2}\right] .
\end{aligned}
$$

The transformations (16)-(18) preserve the domain of definition of the harmonic function $v$, which is very important when considering boundary value problems.

3.2. On the equation $\Delta_{2} u^{*}=e^{-u^{*}}$ An important modification of (11) is the equation

$$
\Delta_{2} u^{*}=e^{-u^{*}},
$$

or $\left(u^{* *}=-u^{*}\right)$ :

$$
\Delta_{2} u^{* *}=-e^{u^{* *}} .
$$

It is easy to see that the equation $\left(27^{\prime}\right)$ is generated by a metric of the form (10), but, in contrast to (11), its curvature must now be positive: $K \equiv+1$. Thus it is convenient to consider an auxiliary metric (20) of curvature $K \equiv+1$ generating, instead of equation (19), the auxiliary equation

$$
y_{\tau \tau}^{* *}+y=0, \quad y^{* *}=y^{* *}(\tau) .
$$


Proceeding as in Section 3.1 we arrive at the following formula relating solutions of (27) to harmonic functions $v$ :

$$
u^{*}(x, t)=\ln \frac{\operatorname{ch}^{2} v}{2\left(v_{x}^{2}+v_{t}^{2}\right)} .
$$

Details are given in [11]

3.3. The construction of a soliton solution for the sine-Gordon equation. Before studing the sine-Gordon equation (7) note that under the reflection $(x, t) \mapsto(x,-t)$ it is transformed to

$$
\bar{u}_{x t}=-\sin \bar{u}
$$

that is, the solutions $u(x, t)$ of the sine-Gordon equation (7) and the solutions $\bar{u}(x, t)$ of the equation (30) are related by

$$
u(x, t)=\bar{u}(x,-t) .
$$

Both the equations (7) and (30) are special cases of the Tchebychef equation (6) generated by the metric (5). In addition, the equation (7) is generated by the metric (5) of constant negative curvature $K(x, t) \equiv-1$, and the equation (31) by the metric of constant positive curvature $K(x, t) \equiv+1$.

To construct a solution $\bar{u}(x, t)$ of $(30)$ let us consider an auxiliary metric of curvature $K \equiv+1$ written in semigeodesic coordinates

$$
d s^{2}=y^{* * 2}(\tau) d \chi^{2}+d \tau^{2}, \quad K(\chi, \tau) \equiv+1 .
$$

The metric (32) generates the equation (28) whose general solution is of the form

$$
y^{* *}(\tau)=A_{1} \sin \tau+A_{2} \cos \tau, \quad A_{1}, A_{2}=\text { const } .
$$

Choose a particular solution $y^{* *}(\tau)=\cos \tau$ and rewrite (32):

$$
d s^{2}=\cos ^{2} \tau d \chi^{2}+d \tau^{2}, \quad K(x, t) \equiv+1 .
$$

The metric (34) of curvature $K \equiv+1$ reduces to the metric (5) of the same curvature under the change of coordinates

$$
x+t=\chi, \quad x-t=\int \frac{d \tau}{\sin \tau} .
$$

The transformation (35) is in a sense an analogue of the transformation (21).

In fact, the metric (34) takes the form

$$
d s^{2}=d x^{2}+2 \cos 2 u(x, t) d x d t+d t^{2} .
$$

Comparing (5) and (36) (under the condition $K \equiv+1$ ) we get a solution of (30):

$$
\bar{u}(x, t)=2 \tau(x, t) .
$$

The function $\tau(x, t)$ is easily found from the second relation (35):

$$
\tau(x, t)=2 \arctan e^{x-t} .
$$


Returning from $(37),(38)$ to $(31)$ we finally obtain a solution $u(x, t)$ of the sine-Gordon equation (7):

$$
u(x, t)=4 \arctan e^{x+t}
$$

The solution (39) is called a "one-soliton" solution of unit amplitude (or a "kink"type solution) of the sine-Gordon equation.

Geometrically it represents the angle of the Tchebychef net formed by the asymptotic lines on a pseudosphere.

4. Generalizations. It should be pointed out that a great number of well-known nonlinear differential equations, having various applications [19], are generated by pseudospherical metrics $[6,21]$ (the metrics of curvature $K \equiv-1$ ). In particular, this is true of the equations of sine-Gordon, Korteweg-de Vries, Burgers, Liouville. One can expect a deep relation between the fundamental equations of mathematical physics and Lobachevsky geometry.

In applications there occur also certain modifications of the equations mentioned above $[2,19]$, corresponding as a rule to the introduction of some correction terms imposed by the specific character of the chosen model of a phenomenon or by the necessity of accounting for new effects (for example, the dissipation).

It seems natural to propose along with the available modification of the nonlinear equations a general geometric approach for obtaining their generalizations. The main feature of the approach is that the original equation and its generalized analogue are both generated by a uniform coordinate net. (For instance, the sine-Gordon equation as well as the Tchebychef equation are generated by the Tchebychef net.) In addition if the curvature of the metric generating the original equation is constant, the metric corresponding to the generalized equation, in general, has the varying curvature $K(x, t)$. Consequently, the generalized equation contains some varying coefficient connected with the curvature. It is important that the given equation and its generalization be investigated in the frame work of the internal geometry global for both of them.

Thus, for constructing a generalized equation it is necessary to consider the curvature of the generating metric (1) whose coefficients (2) in the Gauss formula (3) are variable functions $K=K(x, t)$.

Some generalized (in geometrical sense) nonlinear equations and the corresponding metrics are listed below:

4.1. The Tchebychef equation (the generalized sine-Gordon equation):

$$
u_{x t}=-K(x, t) \sin u(x, t)
$$

This equation is generated by the metric (5) (the Tchebychef net).

4.2. The generalized Korteweg-de Vries equation (KdV):

$$
u_{t}=(1+K(x, t)+6 u) u_{x}+u_{x x x} .
$$


The metric $[6]$ is

$$
\begin{aligned}
d s^{2}= & {\left[(1-u)^{2}+\eta^{2}\right] d x^{2} } \\
& +2\left[(1-u)\left(-u_{x x}+\eta u_{x}-\eta^{2} u-2 u^{2}+\eta^{2}+2 u\right)+\eta\left(\eta^{3}+2 \eta u-2 u_{x}\right)\right] d x d t \\
& +\left[\left(-u_{x x}+\eta u_{x}-\eta^{2} u-2 u^{2}+\eta^{2}+2 u\right)^{2}+\left(\eta^{3}+2 \eta u-2 u_{x}\right)^{2}\right] d t^{2}, \\
\eta= & \text { const }
\end{aligned}
$$

(the $\mathrm{KdV}$-net).

4.3. The generalized modified Korteweg-de Vries equation (MKdV):

$$
u_{t}=\left(1+K(x, t)+\frac{3}{2} u^{2}\right) u_{x}+u_{x x x} .
$$

The MKdV equation is generated by the metric (8) [6] (the MKdV-net).

4.4. The generalized Burgers equation:

$$
u_{t}=(1+K(x, t)+u) u_{x}+u_{x x} .
$$

The corresponding metric [6] has the form

$$
\begin{aligned}
d s^{2}= & \left(\frac{u^{2}}{4}+\eta^{2}\right) d x^{2}+2\left[\frac{\eta^{2} u}{2}+\frac{u}{4}\left(\frac{u^{2}}{2}+u_{x}\right)\right] d x d t \\
& +\left[\left(\frac{u^{2}}{4}+\frac{u_{x}}{2}\right)^{2}+\frac{\eta^{2}}{4} u\right] d t^{2}
\end{aligned}
$$

(the Burgers net).

4.5. The generalized Liouville equation:

a) elliptic:

$$
\Delta_{2} u=-K(x, t) e^{u}
$$

This equation is generated by the metric (10) (the elliptic Liouville net).

b) hyperbolic:

$$
u_{x t}=-K(x, t) e^{u}
$$

The metric $[6]$ is

$$
d s^{2}=\left(u^{2}+\eta^{2}\right) d x^{2}+2 \eta e^{u} d x d t+e^{2 u} d t^{2}
$$

(the hyperbolic Liouville net).

4.6. The generalized sh-Gordon equation:

a) elliptic:

$$
\Delta_{2} u=-K(x, t) \operatorname{sh} u \text {. }
$$

The metric is

b) hyperbolic:

$$
d s^{2}=\operatorname{ch}^{2} \frac{u}{2} d x^{2}+\operatorname{sh}^{2} \frac{u}{2} d t^{2}
$$

$$
u_{x t}=-K(x, t) \operatorname{sh} u \text {. }
$$

The metric [6] is

$$
d s^{2}=\left(u^{2}+\eta^{2}\right) d x^{2}+2 \eta \operatorname{ch} u d x d t+\operatorname{ch}^{2} u d t^{2} .
$$


4.7. The generalized equation generated by the metric (20):

$$
y_{x x}+K(x, t) y(x)=0 .
$$

\section{Discussions of the suggested geometrical concept of differential equations}

5.1. The considered geometrical interpretation of the differential equations connecting them by means of Gauss formula (3) with the special coordinate nets on the twodimensional smooth manifolds may be probably taken as the base for the creation of a new geometrical formalism for partial differential equations (PDE). The perspectives of such a formalism are based on the possibility of applying the instruments and methods of non-Euclidean geometry for the study of PDE. It seems natural to call such a formalism the Gaussian formalism for differential equations.

As can be seen from Section 3 the development of the Gaussian formalism for nonlinear PDE is closely associated with the problem of describing the so-called C-integrable systems $[4,5]$.

5.2. Let us make a brief review of the papers concerning the interpretation of PDE mentioned above.

In $[20,21]$ a striking similarity has been established between the linear eigenvalues problem in the inverse scattering problem [9] and the structural relations for pseudospherical surfaces in $E^{3}$ [10]. In particular, if an equation is integrable via the inverse scattering method then 1 -forms $\omega_{1}, \omega_{2}, \omega_{12}$ determining the pseudospherical surface in $E^{3}$ can be reconstructed according to the data of the direct scattering problem.

The results stated in [20, 21] have been developed in papers $[3,6,18]$. These papers contain a detailed analysis of the structural relations of pseudospherical surfaces in $E^{3}$. In addition, necessary and sufficient conditions for the validity of the discussed geometrical interpretation for vast classes differential equations (first of all, of evolutionary type) are presented.

A similar geometrical approach is proposed in [7, 22].

5.3. The notions of $\mathbf{G}$-class and $\Lambda^{2}$-class of differential equations stated in the present paper rely only on the metric and consequently deal exclusively with internal geometry. The related works mentioned in 5.2 are based on the Cartan exterior forms method and are connected with the investigation of structural equations for surfaces in $E^{3}$, thus involving external geometry. We shall illustrate that the external geometrical interpretation follows from the approach proposed in the present paper.

In fact, let us consider the fundamental equations of surface theory in $E^{3}$ determining the coefficients $L, M, N$ of the second quadratic form of the surface

$$
\begin{gathered}
L_{t}+\Gamma_{11}^{1} M+\Gamma_{11}^{2} N=M_{x}+\Gamma_{12}^{1} L+\Gamma_{12}^{2} M, \\
M_{t}+\Gamma_{12}^{1} M+\Gamma_{12}^{2} N=N_{x}+\Gamma_{22}^{1} L+\Gamma_{22}^{2} M, \\
\frac{L N-M^{2}}{E G-F^{2}}=K .
\end{gathered}
$$

Determining, for example, the coefficient $N=N(L, M, K, E, F, G)$ from (40c) and substituting it in (40a) and (40b) we get a system of two equations for $L, M$ which are 
subjected (for analytic $E, F, G, K$ ) to the Cauchy-Kovalevskaya theorem. Thus, there exists a solution $L, M, N$ of (40a)-(40c) and, consequently, the surface defined by two quadratic forms with the coefficients $E, F, G$, and $L, M, N$, and the Gaussian curvature $K$, realizing the geometrical interpretation of the equation.

In addition, the suggested intrinsic geometrical interpretation of the equations admits the use of different types of metrics (for example, indefinite ones [1]), and its exterior geometrical prolongation has the natural arbitrariness in the choice of the enveloping space.

5.4. The "net-interpretation" of nonlinear PDE lays foundation for the development of numerical methods of their integration. The key question here is that of finding some invariant (characteristic) of the net generating the equation under investigation. Next, the planimetric problem of constructing the net corresponding to the given invariant is solved. And according to the characteristics of the constructed net the solution of PDE is calculated.

5.5. The Gaussian formalism introduced above has some applications in theoretical physics. If some phenomenon or physical process is described by an equation of $\mathbf{G}$-class, the corresponding manifold with the metric generating the given G-equation may be interpreted as the non-Euclidean phase space characterizing the process under consideration $[12,14,15]$. If the curvature of the phase space (metric) is negative, the space inevitably exhibits some singularities (for instance, irregular edges or cusps of pseudospherical surfaces in $E^{3}$ ).

It is very important that the singularities of the phase spaces correspond to the particular (invariant) states of physical systems characterized by minimum energy losses. This interpretation of the phenomena can lead to a geometrical explanation of many physical effects.

6. Problems. Let us formulate the problems connected with the realization of the Gaussian formalism for PDE.

6.1. The problem of reconstructing the generating metric (preferably of constant curvature) corresponding to a given differential equation.

6.2. The problem of the existence of global isometries of spaces having the same curvature and generating different PDE. Or the problem of global transformation of the corresponding coordinate nets.

6.3. The geometrical classification of two-dimensional coordinate nets corresponding to different types of PDE.

6.4. The elaboration of geometrical algorithms for the numerical integration of nonlinear equations (the net approach).

6.5. The development of the concept of non-Euclidean phase space.

6.6. The generalization of the Gaussian formalism to higher dimensions. 


\section{References}

[1] A. Artikbaev and D. D. Sokoloff, Geometry "in the Large" in Plane Space-Time, Fan, Tashkent, 1991 (in Russian).

[2] A. Barone and G. Paterno, The Josephson Effect, Mir, Moscow, 1984 (in Russian).

[3] R. Beals, M. Rabelo and K. Tenenblat, Bäcklund transformation and inverse scattering for some pseudospherical surface equations, Stud. Appl. Math. 81 (1989), 125-151.

[4] F. Calogero and X. Ji, C-integrable partial differential equations I, J. Math. Phys. 32 (4) (1991), 875-887.

[5] - - - C-integrable PDEs II, ibid. 32 (10) (1991), 2703-2717.

[6] S. S. Chern and K. Tenenblat, Pseudospherical surfaces and evolution equations, Stud. Appl. Math. 74 (1) (1986), 55-83.

[7] M. Crampin and D. J. Saunders, The sine-Gordon equation, Tchebyshev nets and harmonic maps, Rep. Math. Phys. 23 (3) (1986), 327-340.

[8] N. Kamran and K. Tenenblat, On differential equations describing pseudospherical surfaces, J. Differential Equations (to be published).

[9] G. L. Lamb, Jr. An Introduction to Soliton Theory, Mir, Moscow, 1983 (in Russian).

[10] V. S. Malakhovskiŭ, An Introduction to the Theory of Exterior Forms, Kaliningrad University Press, Kaliningrad, 1980 (in Russian).

[11] A. G. Popov, Exact formulas for the construction of solutions of the Liouville equation $\Delta_{2} u=e^{u}$ from solutions of the Laplace equation $\Delta_{2} v=0$, Dokl. Akad. Nauk 333 (4) (1993), 440-441 (in Russian).

[12] - The geometrical approach to the interpretation of solutions of the sine-Gordon equation, Dokl. Akad. Nauk SSSR 312 (5) (1990), 1109-1111 (in Russian).

[13] - On the transformation of local solutions of the equations connected with surface geometry, Izv. Vyss. Uchebn. Zaved. Mat. 9 (1993), 1-10 (in Russian).

[14] - The phase spaces of nonzero curvature and evolution of physical systems, Vestnik Moskov. Univ. 34 (6) (1993), 7-13.

[15] E. G. Poznyak and A. G. Popov, The geometry of the sine-Gordon equation, Itogi Nauki i Tekh., Problems in Geometry 23 (1991), 99-130 (in Russian).

[16] - - - The Lobachevsky geometry and equations of mathematical physics, Dokl. Akad. Nauk 332 (4) (1993), 418-421 (in Russian).

[17] E. G. Poznyak and E. V. Shikin, Differential Geometry, Moscow University Press, Moscow, 1990 (in Russian).

[18] M. Rabelo, On evolution equations which describe pseudospherical surface, Stud. Appl. Math. 81 (1989), 221-248.

[19] A. Sanchez and L. Vazquez, Nonlinear wave propagation in disorded media, Internet. J. Modern Phys. B 5 (18) (1991), 2825-2882.

[20] R. Sasaki, Geometrization of soliton equations, Phys. Lett. A 71 (1979), 390-392.

[21] —, Soliton equations and pseudospherical surfaces, Nucl. Phys. B 154 (1979), 343-357.

[22] A. Sym, Soliton surfaces, Lett. Nuovo Cimento 33 (12) (1982), 394-400.

[23] P. Tchebychef [P. Chebyshev], Sur la coupe des vêtements, Assoc. franç. pour l'avancement des sciences, 1878; see also Euvres II. 